\title{
A Microemulsion for the Oral Drug Delivery of Pitavastatin
}

\author{
Evren Gundogdu ${ }^{1 *}$, Yucel Baspinar ${ }^{2}$, Cinel Koksal ${ }^{2,3}$, Iskender Ince ${ }^{2}$ and Ercument Karasulu ${ }^{1,2}$ \\ ${ }^{1}$ Faculty of Pharmacy, Department of Biopharmaceutics and Pharmacokinetics, Ege University, 35100 Bornova, Izmir, Turkey \\ ${ }^{2}$ Center for Drug Research and Development and Pharmacokinetic Applications, Ege University, 35100, Izmir, Turkey \\ ${ }^{3}$ Faculty of Science, Department of Biology, Ege University, 35100 Bornova, Izmir, Turkey
}

\begin{abstract}
The aim of this study was to evaluate the potential application of a microemulsion as an oral drug delivery system for pitavastatin and to compare its in vitro cytotoxicity with a pitavastatin solution. The here developed water in oil (w/o) microemulsion system was optimized by using pseudo ternary phase diagrams, composed of span 80 , Lutrol F 127, isopropyl alcohol, oleic acid and distilled water. This microemulsion was characterized according to its phase behavior, droplet size, viscosity, conductivity, refractive index and polydispersity index. The final concentration of pitavastatin in the microemulsion was $1 \mathrm{mg} / \mathrm{ml}$. Moreover, the in vitro cytotoxicity studies were performed with Caco2 and MCF-7 cell lines for the microemulsion and the pitavastatin solution. The in vitro cytotoxicity studies revealed no cytotoxic effect on Caco-2 and MCF-7 cells for both microemulsions, with and without pitavastatin and for the pitavastatin solutions. In conclusion, the microemulsion formulation may be used as an effective and alternative drug delivery system for the hyperlipidemic oral therapy with pitavastatin.
\end{abstract}

Keywords: Microemulsion; Pitavastatin; Cytotoxicity; Drug delivery system

\section{Introduction}

3-Hydroxy-3-methylglutaryl coenzyme A (HMG-CoA) reductase inhibitors, more commonly known as the statins, are the most commonly prescribed lipid-modifying drugs [1,2]. Competitive inhibition of HMG-CoA reductase by the statins decreases hepatocyte cholesterol synthesis, which results in an increased extraction of LDL-C from the blood and decreased circulating of the LDL-C concentration $[3,4]$.

Pitavastatin (PT), (+)-monocalcium bis $\quad(3 \mathrm{R}, 5 \mathrm{~S}, 6 \mathrm{E})-7-(2-$ cyclopropyl-4-[4-fluorophenyl]-3-quinolyl-3,5-dihydroxy-6heptanoate), is a potent synthetic inhibitor of HMG-CoA reductase and was developed for the treatment of hypercholesterolaemia. It can reduce plasma levels of LDL cholesterol by $40 \%$ in hypercholesterolaemic patients $[5,6]$. In humans, PT is only minimally metabolized by the cytochrome P450 2C9 isoenzyme. The major metabolic pathway of PT involves its initial glucuronidation by uridine diphosphateglucuronosyltransferase and then spontaneous lactonization by the elimination of the glucuronide moiety. Moreover, the lactone form can be reversibly converted to the parent drug [6]. PT is excreted predominantly into the bile and thereby enters the enterohepatic circulation. Very little parent drug is excreted into the urine $[7,8]$.

Microemulsions (MEs) have recently attracted much attention in pharmaceutical research areas [9]. High thermodynamic and kinetic stability, low viscosity and optical transparency make them very attractive as a pharmaceutical application form to improve the solubility, the dissolution and the oral absorption of poorly water-soluble drugs [10]. Further advantages of using MEs as drug delivery systems include a better drug solubilization and the protection against enzymatic hydrolysis, as well as the potential for an enhanced absorption due to a surfactant-induced improvement in the permeability. In addition, MEs represent an interesting and potentially quite powerful alternative carrier system for drug delivery because of their high solubilization capacity, transparency, ease of preparation, and high diffusion and absorption rates, compared to solvents without the surfactant system $[11,12]$. MEs are forming spontaneously and are composed of surfactant $(\mathrm{S})$, co-surfactant $(\mathrm{CoS})$, oil and water with a particle size of less than $100 \mathrm{~nm}$ in diameter. The combination of surfactants with oils to form MEs offers an advantage with a low free energy and a large surface area, which were considered to be responsible for transporting drugs to gastrointestinal membrane for absorption $[13,14]$.

\section{Methods}

\section{Materials}

PT was provided by Basel Drug Company (Istanbul, Turkey). Diphenhydramine was purchased from Sigma Chemical Co (St Louis, MO). Lutrol F 127 was purchased from BASF (Ludwigshafen, Germany). Span 80, oleic acid, and isopropyl alcohol were obtained from Sigma- Aldrich (St Louis, MO). Cell culture reagents and supplies were obtained from Gibco Invitrogen (Grand Island, NY).

\section{Preparation of the microemulsions}

In order to find out the concentration range of the components for the existing range of the ME, pseudo-ternary phase diagrams were constructed using the water titration method at an ambient temperature. Oleic acid was selected as the oil phase. The effects of the Ss (mixtures of span 80 and lutrol F 127 at w/w ratios of 9.5:0.5 with a hydrophilic-lipophilic balance (HLB) of 5.53 and of the CoS (isopropyl alcohol) on the pseudoternary phase diagram were systematically observed at room temperature. For each phase diagram at a specific S/ $\mathrm{CoS}$ weight ratio, the ratios of oil to the mixture of $\mathrm{S} / \mathrm{CoS}$ were varied as, 1:1, 2:1, 3:1, 4:1 and 5:1 (w/w). Lutrol F 127 were melted at $50^{\circ} \mathrm{C}-60^{\circ} \mathrm{C}$

*Corresponding author: Evren Gundogdu, Faculty Of Pharmacy, Department Of Biopharmaceutics and Pharmacokinetics, Ege University, 35100 Bornova, Izmir Turkey, E-mail: evren.gundogdu@ege.edu.tr

Received January 11, 2013; Accepted January 28, 2013; Published January 30 2013

Citation: Gundogdu E, Baspinar Y, Koksal C, Ince I, Karasulu E (2013) A Microemulsion for the Oral Drug Delivery of Pitavastatin. Pharmaceut Anal Acta 4 209. doi:10.4172/2153-2435.1000209

Copyright: (c) 2013 Gundogdu E, et al. This is an open-access article distributed under the terms of the Creative Commons Attribution License, which permits unrestricted use, distribution, and reproduction in any medium, provided the original author and source are credited. 
and blended with span 80 to make the S mixture. Afterwards, the oil phase and the $S$ mixture were mixed. These mixtures were titrated, drop-by-drop, with distilled water while being gently stired at $25 \pm 1^{\circ} \mathrm{C}$ until. The appearances from clear to turbid and turbid to clear were investigated, respectively. After being equilibrated, the mixtures were assessed by visual characterization (Figures 1A-E) $[15,16]$.

After the identification of ME region in the phase diagrams, the ME formulation was selected with the desired component ratios. The blank and the PT containing ME were prepared by the same way. The PTincorporating ME was prepared by dissolving the drug powder into the ME system. The final concentration of PT in ME system was $1 \mathrm{mg} / \mathrm{mL}$.

\section{Characterization of the microemulsions}

The ME was analyzed for various properties. The average droplet size and the polydispersity index (PDI) of the ME in the presence and absence of PT were measured by photon correlation spectroscopy (Nano ZS, Malvern Instruments, UK). The viscosities of the MEs in the presence and absence of PT were detected at $25.0 \pm 1^{\circ} \mathrm{C}$ using a Brookfield digital viscometer-III rheometer V 3.3 HB (Middleboro, MA) (Spindle: SC4-21). The refractive index and the electric conductivity of the ME in the presence and absence of $\mathrm{PT}$ were measured using a refractometer (Atago RX-7000 CX, Japan) at $25 \pm 1{ }^{\circ} \mathrm{C}$ and monitored quantitatively by using a conductometer and its conductometer probe (Jenway 4071, UK) at $25 \pm 1^{\circ} \mathrm{C}$, respectively.

\section{Stability of the microemulsion}

To evaluate the stability of the optimized ME formulation of PT, the formulation was added into sealed glass vials which were stored at $25 \pm 1^{\circ} \mathrm{C}$ and $40 \pm 1^{\circ} \mathrm{C}$ in climate cabin for 6 months. The clarity and droplet size were investigated at predetermined intervals $(n=6)$.

\section{Cytotoxicity studies of the pitavastatin solution}

The Caco-2 and MCF-7 cells were obtained from American Type Culture Collection (ATCC) and used for the cytotoxicity tests. The experimental cells were counted in a hemocytometer (Reichert Co., USA) using the trypan blue exclusion method. The cells were plated on 96-well flat-bottomed plates, with each well at a density of $1 \times 10^{4}$ cells, and incubated for $24 \mathrm{~h}$ at $37^{\circ} \mathrm{C}$ in the $\mathrm{CO}_{2}$ incubator. After the cells were attached on 96-well flat-bottomed plates, $100 \mu \mathrm{L}$ of PT solutions in Hank's Balanced Salt Solution (HBSS) at different concentrations (100 to $2000 \mu \mathrm{g} / \mathrm{mL}$ ) were directly added to plates. The cells were incubated with the PT solution for the measurement duration of 1,2 and 3 days, at $37^{\circ} \mathrm{C}$ in the $\mathrm{CO}_{2}$ incubator. The cytotoxicity following the above-mentioned treatments was evaluated by 3-[4,5-dimethylthiazol2-yl]-3, 5-diphenyltetrazolium bromide dye (MTT) assay. Briefly, at the end of the incubation period the culture medium was aspirated and cells were washed with phosphate buffer solution (PBS) $(\mathrm{pH}=7.4)$. Then cells were incubated with $100 \mu \mathrm{l}$ of the MTT solution $(0.5 \mathrm{mg} /$ $\mathrm{mL}$ ) in Dulbecco Modified Eagle Medium (DMEM) without Fetal Bovine Serum (FBS) for $4 \mathrm{~h}$ at $37^{\circ} \mathrm{C}$. The MTT solution with culture medium was removed without a formation of the formazan crystals. $100 \mu \mathrm{l}$ of dimethyl sulfoxide (DMSO) was added in order to dissolve the formed formazan crystals. After solubilizing the crystals, the absorbance was measured with the ELISA microplate reader (Thermo vario scan-FHA multiplate reader) at a wavelength of $570 \mathrm{~nm}$. The

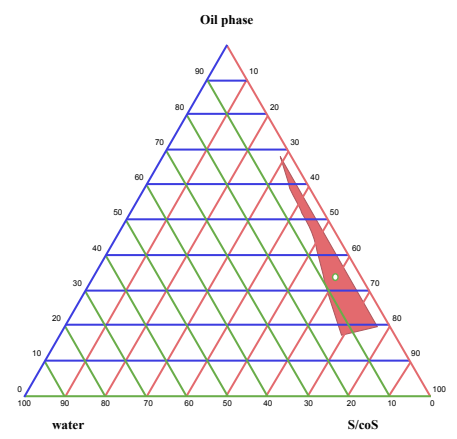

(A)

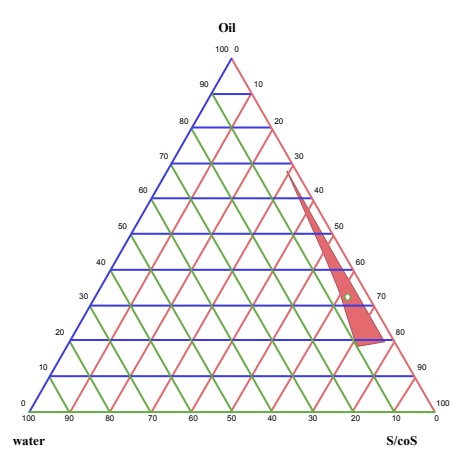

(B)

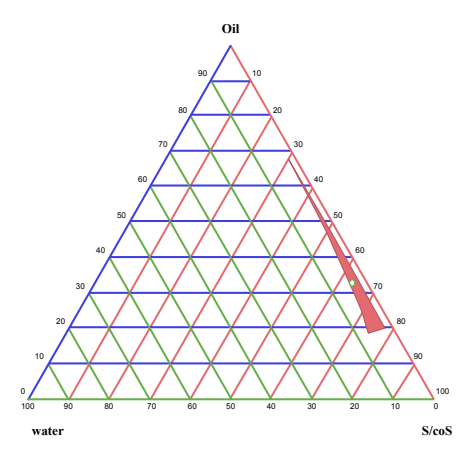

(C)

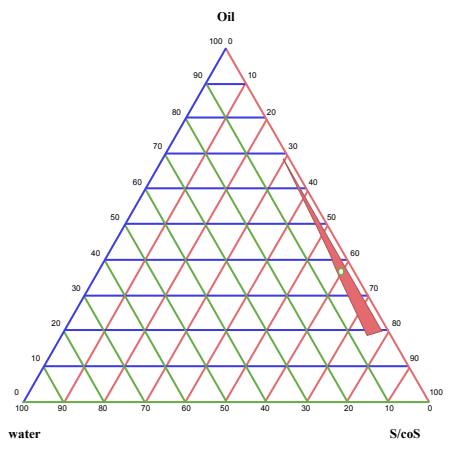

(D)

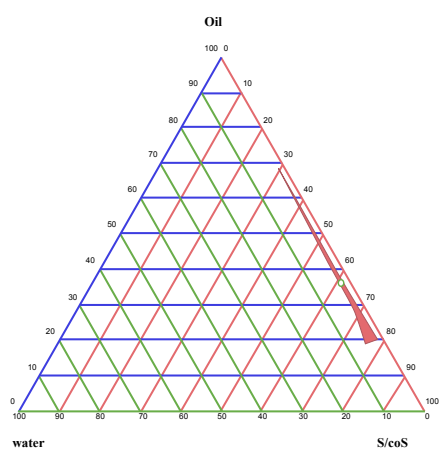

(E)

Figure 1: Pseudo-ternary phase diagrams of the ME system. (A) 1:1 S/CoS ratio, (B) 2:1 S/CoS ratio, (C) 3:1 S/CoS ratio, (D) 4:1 S/CoS ratio, (E) 5:1 S/CoS ratio. 
results of the experiments were evaluated due to the cell viability (\%). All experiments were performed in triplicate.

$$
\text { Cell viability }(\%)=\mathrm{T} / \mathrm{C} \times 100(\text { Equation } 1)
$$

Where $\mathrm{T} / \mathrm{C}$ is the test treatment over control $(\mathrm{T} / \mathrm{C})$ values $[17,18]$.

\section{Cytotoxicity studies of the pitavastatin microemulsion}

The Caco- 2 and the MCF-7 cells were also used for the cytotoxicity tests of the microemulsions. The cells were plated on 96-well flatbottomed plates, with each well at a density of $1 \times 10^{4} \mathrm{cells}$, and incubated for $24 \mathrm{~h}$ at $37^{\circ} \mathrm{C}$ in the $\mathrm{CO}_{2}$ incubator. After the cells were attached on 96-well flat-bottomed plates, ME in the absence and presence of PT at a desired concentration $(1000 \mu \mathrm{g} / \mathrm{ml})$ were directly added to the cell culture medium of $100 \mu \mathrm{l}$. The cells were incubated with the ME for the measurement duration of 1,2 and 3 days, at $37^{\circ} \mathrm{C}$ in the $\mathrm{CO}_{2}$ incubator. The cytotoxicity following the above-mentioned treatments was evaluated by the MTT assay.

\section{HPLC analysis of pitavastatin}

The HPLC analysis method was modified from previous studies [7]. The samples were analyzed by using a HPLC system (The HPLC system was equipped with a Agilent 1100 series, a reverse phase ACE $5 \mathrm{C}_{18}$ column $(150 \mathrm{~mm} \times 4.6 \mathrm{~mm}, 5 \mu \mathrm{m}$, ACT, Scotland) $)$ that has a UV spectroscopic detector. The mixture of methanol, distilled water and formic acid solutions (75:25:0.05 v/v/v) was used as the mobile phase at a flow rate of $0.8 \mathrm{ml} / \mathrm{min}$. The injection volume was $20 \mu \mathrm{l}$. The wavelength of the DAD detector was set as $254 \mathrm{~nm}$. All samples were filtrated through membrane filter $(0.2 \mu \mathrm{m}$ Nylon, Millipore Millex-GN) before injection. The peak area correlated linearly with PT concentration in the range of $5-250 \mu \mathrm{g} / \mathrm{ml}$ with the lowest detection limit at $0.5 \mu \mathrm{g} / \mathrm{ml}$, and the average correlation coefficient was 0.999 .

\section{Statistical data analysis}

Statistical analysis was performed using one way analysis of variance (ANOVA) to evaluate differences between the ME and the solution, both containing PT. The data were considered as statistically significant at $\mathrm{p}<0.05$.

\section{Results}

\section{Construction of the pseudo ternary phase diagrams}

Investigation of the phase behavior of these systems demonstrated that our approach was suitable for determining the water phase, the oil phase, the $S$ concentration, and the CoS concentration at which the transparent ME system was formed. The construction of a phase diagram makes it easy to identify the concentration range of the components in the MEs. Figure 1 shows the phase diagrams constructed to determine the optimum S/CoS concentration ratio for the formulation of a w/o ME consisting of span 80, lutrol F 127, oleic acid, isopropyl alcohol, and water. The S/CoS and S mixture (span 80/ lutrol F 127) ratios were found to be 1:1 and 9.5:0.5 for the optimized
ME. As shown in figure 1A, the area of w/o ME becomes enlarged and is highest at an $\mathrm{S} / \mathrm{CoS}$ ratio of $1: 1$. The exact composition according to oil, $\mathrm{S}, \mathrm{CoS}$, and aqueous phases is shown in table 1 .

\section{Characterization of the microemulsion}

The characteristic parameters of the ME were shown in table 2. The particle size analysis showed that the mean droplet size of the ME in the presence and absence of PT was between $39.05 \pm$ 0.87 and $33.29 \pm 1.76 \mathrm{~nm}$ and the PDIs were 0.044 for both MEs, with and without PT. When PT was loaded to the ME system, the formulation showed no change in its transparency, but the mean droplet size of ME slightly decreased from $39 \mathrm{~nm}$ to $33 \mathrm{~nm}$ (Table 2). The possible reason might be that loading the ME with PT affects the density of the formulation and thus reflects the particle size. When PT was dissolved and dispersed into the emulsifying membrane layer (composed of $\mathrm{S}$ and $\mathrm{CoS}$ ) and oil phase, the chemical groups in PT could react with the other groups of the $S$, the $\mathrm{CoS}$ and the oil phase by forming hydrogen bonds. This may be because of the decreased surface tension due to the presence of the $S$ and the CoS [19]. The PDI value is describing the width of the particle size distribution of the formulation. All PDI values were smaller than 0.2 , which shows a narrow distribution of the particle size.

It was described in the literature, that a $\mathrm{CoS}$ free viscous ME was showing a pseudo-plastic behavior. The less viscous CoS containing preparations had Newtonian flow. Therefore, incorporating the $\mathrm{CoS}$ into the MEs resulted in a significant viscosity reduction of the formulations, along with a flow change to a simple Newtonian behavior $[16,20]$. In our study, the viscosity of the MEs (with and without) were nearly close to each other, namely $104.7 \pm 0.15 \mathrm{cP}$ (blank ME) and 103.8 $\pm 0.25 \mathrm{cP}$ (PT loaded ME) ( $>>0.05)$ (Table 2). The refractive indexes of both ME formulations ranged from $1.412 \pm 0.12$ (blank ME) to $1.415 \pm$ 0.21 (PT loaded ME). When PT was loaded into ME, refractive index of ME was increased (Table 2). The electrical conductivity was about 1.1 $\mu \mathrm{s} / \mathrm{cm}$ for both ME formulations (Table 2).

\section{Stability of the microemulsion}

After storage of the PT loaded ME at $25 \pm 1{ }^{\circ} \mathrm{C}$ and $40 \pm 1^{\circ} \mathrm{C}$ for 6 months, the ME was still clear and transparent without any phase separation. The droplet size did not changed significantly $(p>0.05)$ (Table 3). It was suggested that the PT incorporated ME was stable during these storage time of 6 months.

\section{Cytotoxicity studies of the pitavastatin solution}

In this study, the effect of PT at different concentrations on Caco-2 cells and MCF-7 cells line has been showed by the MTT test. The cell viabilities (Figure 2) after the application of the PT solutions were found to be between $98.37 \pm 5.86 \%$ and $99.99 \pm 5.75 \%$ for Caco-2 cells after $72 \mathrm{~h}$ at different concentrations. This demonstrates that the PT solution was not toxic on Caco-2 cells (Figure 2A). In addition, after exposure to $\mathrm{PT}$ at different concentrations for $72 \mathrm{~h}$, the cytotoxic effect on MCF-7 was also investigated by using the MTT assay. The cell viabilities after

\begin{tabular}{|c|c|c|c|c|}
\hline Formulation & Oil (\%) & S 1(\%) & S 2 (\%) & CoS (\%) \\
\hline Microemulsion (ME) & 34.05 & 1.49 & 28.31 & 29.79 \\
\hline
\end{tabular}

Table 1: The contents of the optimized ME formulation (S1- Lutrol F 127, S2- Span 80, CoS- isopropyl alcohol).

\begin{tabular}{|c|c|c|c|c|c|c|}
\hline & Electrical conductivity $(\mu \mathrm{S} / \mathrm{cm})$ & Viscosity (cP) & Particle size (nm) & PDI & Refractive index & PT Content (mg/mL) \\
\hline ME & $1.1 \pm 0.03$ & $104.7 \pm 0.15$ & $39.05 \pm 0.87$ & $0.044 \pm 0.01$ & $1.412 \pm 0.12$ & - \\
\hline ME-PT & $1.1 \pm 0.02$ & $103.8 \pm 0.25$ & $33.29 \pm 1.76$ & $0.044 \pm 0.02$ & $1.415 \pm 0.21$ & $1 \pm 0.17$ \\
\hline
\end{tabular}

Table 2: Characterization of the microemulsions (ME is a blank microemulsion, ME-PT is a microemulsion with PT). 
application of the PT solutions were found between $98.04 \pm 6.53 \%$ and $98.74 \pm 9.41 \%$ for the MCF-7 cells (Figure 2B). Evaluation of the results revealed that PT was not cytotoxic for these cell lines during the test period (Figure 2B).

\section{Cytotoxicity studies of the pitavastatin loaded microemulsion}

The cytotoxicity results of PT loaded ME was shown in figures $3 \mathrm{~A}$ and $3 \mathrm{~B}$, expressed as the cell viability \%. The concentration of PT $(1000 \mu \mathrm{g} / \mathrm{mL})$ in the MEs caused no cytotoxicity on the Caco- 2 cell line (Figure 3A) and on the MCF-7 cell line (Figure 3B). When the ME with and without PT was applied to the cell culture medium, the samples did not show any cytotoxicity on the MCF-7 cells line during the test period. Furthermore, the ME formulation containing PT did not affect the MCF-7 cells line growth and survival during the direct and continuous exposure to the cell for the test period. The cell viability of MCF-7 was found to be between $99.62 \pm 2.27 \%$ and $98.91 \pm 2.49 \%$ for both MEs during $72 \mathrm{~h}$. According to the in vitro cytotoxicity studies, it was concluded that there was no cytotoxic effect on Caco-2 cell and MCF-7 for both ME formulations with and without PT (ME and MEPT).

\begin{tabular}{|c|c|c|}
\hline \multicolumn{3}{|c|}{ Temperature } \\
\hline \multirow{2}{*}{ Month } & $\mathbf{2 5}^{\circ} \mathbf{C}$ & $\mathbf{4 0}^{\circ} \mathbf{C}$ \\
\cline { 2 - 3 } & Droplet size & Droplet size \\
\hline 0 & $33.29 \pm 0.95$ & $33.29 \pm 0.95$ \\
\hline 3 & $33.72 \pm 0.61$ & $34.01 \pm 0.55$ \\
\hline 6 & $33.34 \pm 0.44$ & $34.72 \pm 0.85$ \\
\hline
\end{tabular}

Table 3: Stability test results of the PT loaded microemulsion (ME-PT).

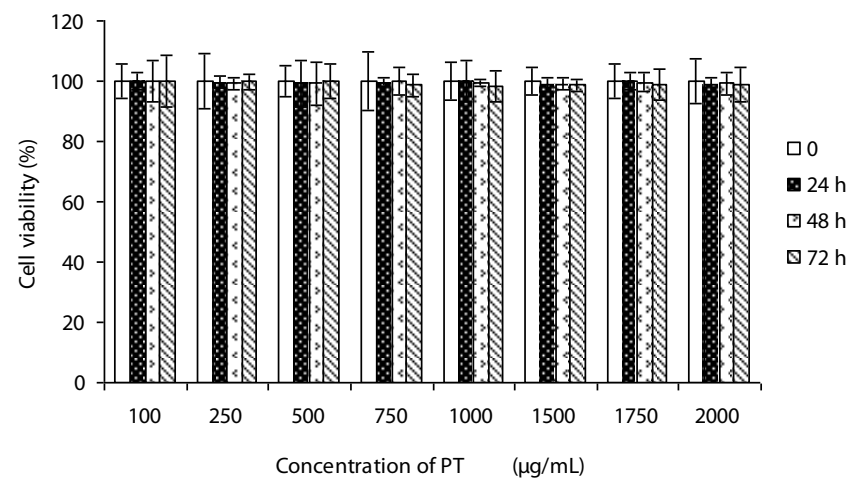

Figure 2A: The cell viabilities of the pitavastatin solution on Caco-2 (A) cells.

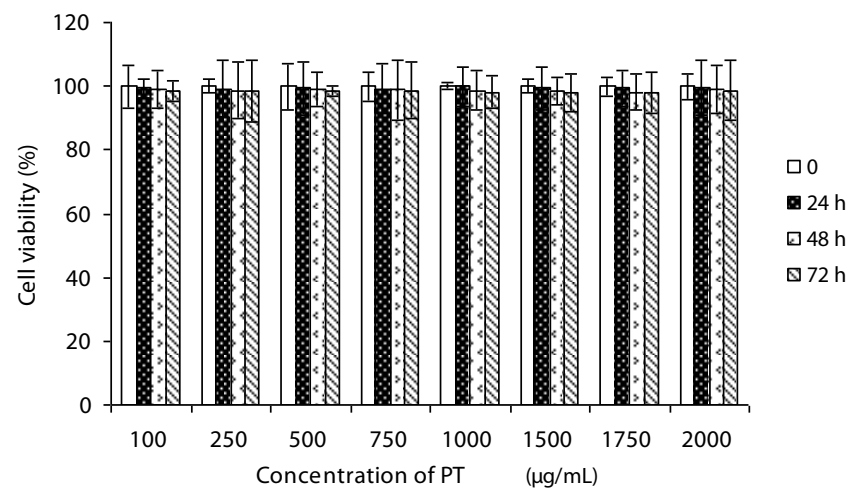

Figure 2B: The cell viabilities of the pitavastatin solution on MCF-7 cells.

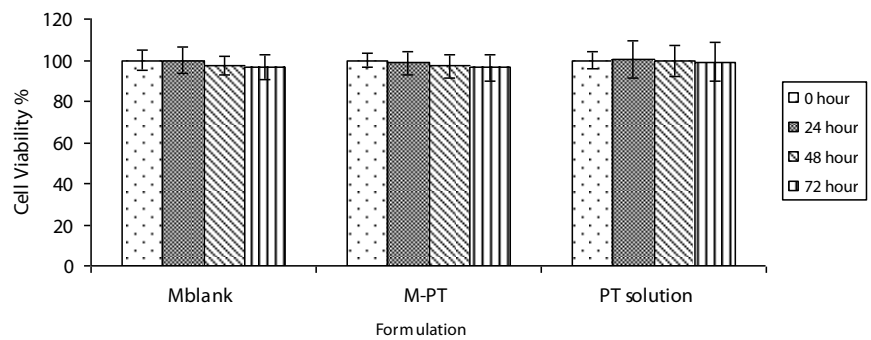

Figure 3A: The cell viabilities of the pitavastatin microemulsion on Caco-2 cells.

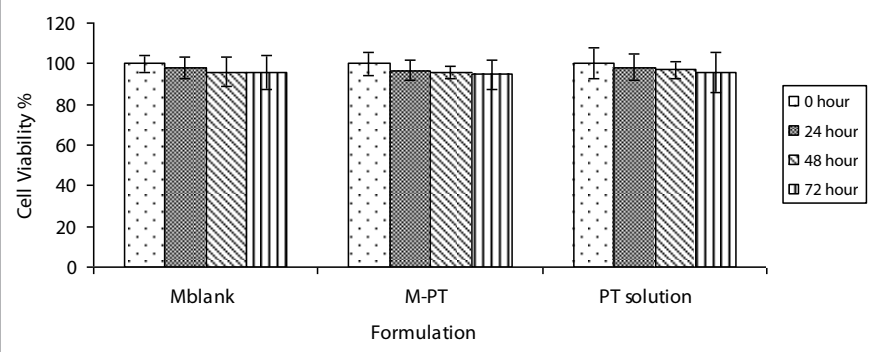

Figure 3B: The cell viabilities of the pitavastatin microemulsion on MCF-7 cells.

\section{Conclusion}

- Recently, many studies have been performed by using ME systems for hyperlipidemic therapy and there are only a few studies about formulations which have been developed for the application of PT. In this study, a new w/o ME system for the oral delivery of PT was developed through the construction of pseudo-ternary phase diagrams and optimization with a simple method. According to the physicochemical characterization and the in vitro cytotoxicity studies, it can be concluded that PT may be incorporated into the ME formulation which is a new drug carrier system,

- The ME formulations (ME and ME-PT) were not cytotoxic for Caco-2 and MCF-7 cell lines.

These results indicate that, this ME is a promising formulation for the alternative oral drug delivery of PT with hyperlipidaemia as the indication.

\section{References}

1. Havel RJ, Rapaport E (1995) Management of Primary Hyperlipidemia. N Eng J Med 332: 1491-1498.

2. Ibuki C, Seino Y, Otsuka T, Kimata N, Inami T, et al. (2012) Switching to Pitavastatin in Statin-Treated Low HDL-C Patients Further Improves the Lipid Profile and Attenuates Minute Myocardial Damage. J Clin Med Res 4: 385-392.

3. Maron DJ, Fazio S, Linton MF (2000) Current Perspectives on Statins Circulation 101: 207-213.

4. Tani S, Nagao K, Hirayama A (2012) Association of cholesteryl ester transfer protein mass with peripheral leukocyte count following statin therapy: a pilot study. Am J Cardiovasc Drugs 12: 349-354.

5. Saito $\mathrm{Y}$, Yamada N, Teramoto T, Itakura H, Hata Y, et al. (2002) A randomized, double-blind trial comparing the efficacy and safety of pitavastatin versus pravastatin in patients with primary hypercholesterolemia. Atherosclerosis 162 : 373-379.

6. Oh ES, Kim CO, Cho SK, Park MS, Chung JY (2012) Impact of ABCC2, ABCG2 and SLCO1B1 polymorphisms on the pharmacokinetics of pitavastatin in humans. Drug Metab Pharmacokinet. 
Citation: Gundogdu E, Baspinar Y, Koksal C, Ince I, Karasulu E (2013) A Microemulsion for the Oral Drug Delivery of Pitavastatin. Pharmaceut Anal Acta 4: 209. doi:10.4172/2153-2435.1000209

Page 5 of 5

7. Tian L, Huang Y, Jia Y, Hua L, Li Y (2008) Development and validation of a liquid chromatography-tandem mass spectrometric assay for pitavastatin and its lactone in human plasma and urine. J Chromatogr B Analyt Technol Biomed Life Sci 865: 127-132

8. Hayashi T, Yokote K, Saito Y, Iguchi A (2007) Pitavastatin: efficacy and safety in intensive lipid lowering. Expert Opin Pharmacother 8: 2315-2327.

9. Gan L, Gan Y, Zhu C, Zhang X, Zhu J (2009) Novel microemulsion in situ electrolyte-triggered gelling system for ophthalmic delivery of lipophilic cyclosporine A: in vitro and in vivo results. Int J Pharm 365: 143-149.

10. Tsai YH, Hsieh YH, Huang YB, Chang JS, Huang CT, et al. (2010) Microemulsions for intravesical delivery of gemcitabine. Chem Pharm Bull 58: 1461-1465.

11. Jadhav KR, Shaikh IM, Ambade KW, Kadam VJ (2006) Applications of microemulsion based drug delivery system. Curr Drug Deliv 3: 267-273.

12. Yin YM, Cui FD, Mu CF, Choi MK, Kim JS, et al. (2009) Docetaxel microemulsion for enhanced oral bioavailability: preparation and in vitro and in vivo evaluation J Control Release 140: 86-94.

13. Zhang YT, Huang ZB, Zhang SJ, Zhao JH, Wang Z, et al. (2012) In vitro cellular uptake of evodiamine and rutaecarpine using a microemulsion. Int $J$ Nanomedicine 7: 2465-2472.
14. $\mathrm{Lu} \mathrm{CH}, \mathrm{Wu} \mathrm{WH}$, Kale RB (2008) Microemulsion-mediated hydrothermal synthesis of photocatalytic TiO2 powders. J Hazard Mater 154: 649-654

15. Gundogdu E, Alvarez Gonzalez IG, Karasulu E (2011) Improvement of effect of water in oil microemulsion as an oral delivery system for fexofenadine: in vitro and in vivo studies. Int J Nanomed 6: 1631-1640.

16. Okur N, Apaydın S, Karabay Yavasoglu NU, Yavasoglu A, Karasulu HY (2011) Evaluation of skin permeation and anti-inflammatory and analgesic effects of new naproxen microemulsion formulations. Int J Pharm 416: 136-144.

17. David M (1997) Studies of cell function, Coder Current Protocols in Cytometry.

18. Fischer D, Li Y, Ahlemeyer B, Krieglstein J, Kissel T (2003) In vitro cytotoxicity testing of polycations: influence of polymer structure on cell viability and hemolysis. Biomaterials 24: 1121-1131.

19. Cui J, Yu B, Zhao Y, Zhu W, Li H, Lou H, et al. (2009) Enhancement of oral absorption of curcumin by self-microemulsifying drug delivery systems. Int $J$ Pharm 371: 148-155.

20. El Maghraby GM (2008) Transdermal delivery of hydrocortisone from eucalyptus oil microemulsion: Effects of cosurfactants. Int J Pharm 355: 285 292. 